\title{
HEART REACTION OF MALE AND FEMALE WITH STREPTOZOTOCIN INDUCED DIABETES ON CHOLINERGIC IMPACTS
}

DOI: 10.36740/WLek202002103

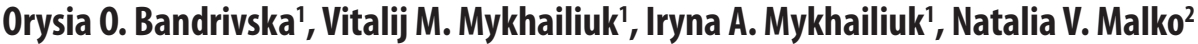 \\ 'I. HORBACHEVSKY TERNOPIL STATE MEDICAL UNIVERSITY, TERNOPIL, UKRAINE \\ 2DANYLO HALYTSKY LVIV NATIONAL MEDICAL UNIVERSITY, LVIV, UKRAINE
}

\begin{abstract}
The aim: Find out the nature of the changes sensitivity of the heart to the cholinergic influences the development of diabetes depending on gender. Materials and methods: In experiments on adult male and female rats, type 1 diabetes by momentary introduction of streptozotocin at a dose of $60 \mathrm{mg} / \mathrm{kg}$ into the peritoneal cavity was modeled. Animals were examined after 15, 30,60 and 90 days after introduction of streptozotocin. We studied the sensitivity of cholinoreceptors of sinus node by the intensity of bradycardia with intravenous injection of acetylcholine and electrical stimulation of the peripheral segment of the right vagus nerve.

Results: It was established that the intensity of the negative chronotropic effect of heart of males by electric stimulation of the vagus nerve, which depends on the reserves of acetylcholine in the presynaptic section and sensitivity of postsynaptic cholinoreceptors, increased gradually and after 90 days prevailed the output value in 2.2 times. This occurred at the background of a slight (10\%) decrease in response to exogenous acetylcholine that reflected the decrease in sensitivity of cholinoreceptors. In females the maximum increase (in 4.4 times) of the intensity of the negative chronotropic effect of vagus nerve stimulation was observed after 30 days from the start of the experiment. After 90 days, this indicator prevailed the controlled one in 3.3 times, which occurred at the background of heart response decreasing to exogenous acetylcholine (in 1.7 times). Conclusions: Obtained results suggest a more significant change in state of cholinoceptor system of myocardium of females compared with males in the dynamics of development of streptozotocin-induced diabetes.
\end{abstract}

KEY WORDS: diabetes mellitus, heart, sex, cholinoreceptors

Wiad Lek. 2020;73(2):224-228

\section{INTRODUCTION}

Diabetes mellitus is one of the leading medical and social problems that ranks third in the world after cardiovascular and oncological diseases in terms of morbidity and the number of complications that lead to disability and death $[1,2]$. Among the causes, cardiovascular disease predominates. According to WHO's latest estimates, more than 200 million people around the world suffer from this disease, which has allowed it to be recognized as a manifestation of the 21 st century non-communicable epidemic with a tendency to spread, which is associated with an increase in age[3].

Analysis of statistical data on the epidemiology of diabetes suggests the presence of a gender component, which suggests the participation of sex hormones in the development of endocrinopathy. It is established that type 2 diabetes in girls occurs more often than in boys[4].

The subject of attention of many researchers in the etiology and pathogenesis of diabetes is myocardial dystrophy, which often causes a deterioration in the state of patients and an increased risk of death[5,6]. Despite the initiation of the development of diabetic cardiomyopathy with chronic hyperglycemia, an important role in its pathogenesis plays a complex of metabolic disorders, including energy imbalance, accumulation in fatty acid cardiomyocytes, oxidative stress, violation of ion exchange mechanisms, accumulation of calcium ions[7].

The manifestations of cardiomyopathy are disturbances of the pumping function of the heart - diastolic left ventricular dysfunction and later systolic, the basis for the development of which is not only the above-mentioned violations, but also autonomous cardiac neuropathy[9]. Its development is leading to the progression of heart failure and is characterized by a disturbance in the balance between sympathetic and parasympathetic regulatory effects on the heart, a decrease in heart rate variability, heart rate adaptation to stress, arrhythmias (sometimes fatal), and a decrease in pumping function of the heart[10].

Despite a large number of scientific data reflecting this problem, experimental studies that show the results of studying the peculiarities of the development and manifestation of diabetes mellitus, depending on sex, are small, and the available ones do not answer a large number of questions. Taking into account available in available scientific sources data on the essential difference in the cholinergic regulation of the heart, depending on sex, it can be assumed that this factor will determine not only the characteristics of the course of diabetes, but also the nature of the cholinergic regulation of the heart[11]. Little research is devoted to the study of the nature of metabolic 

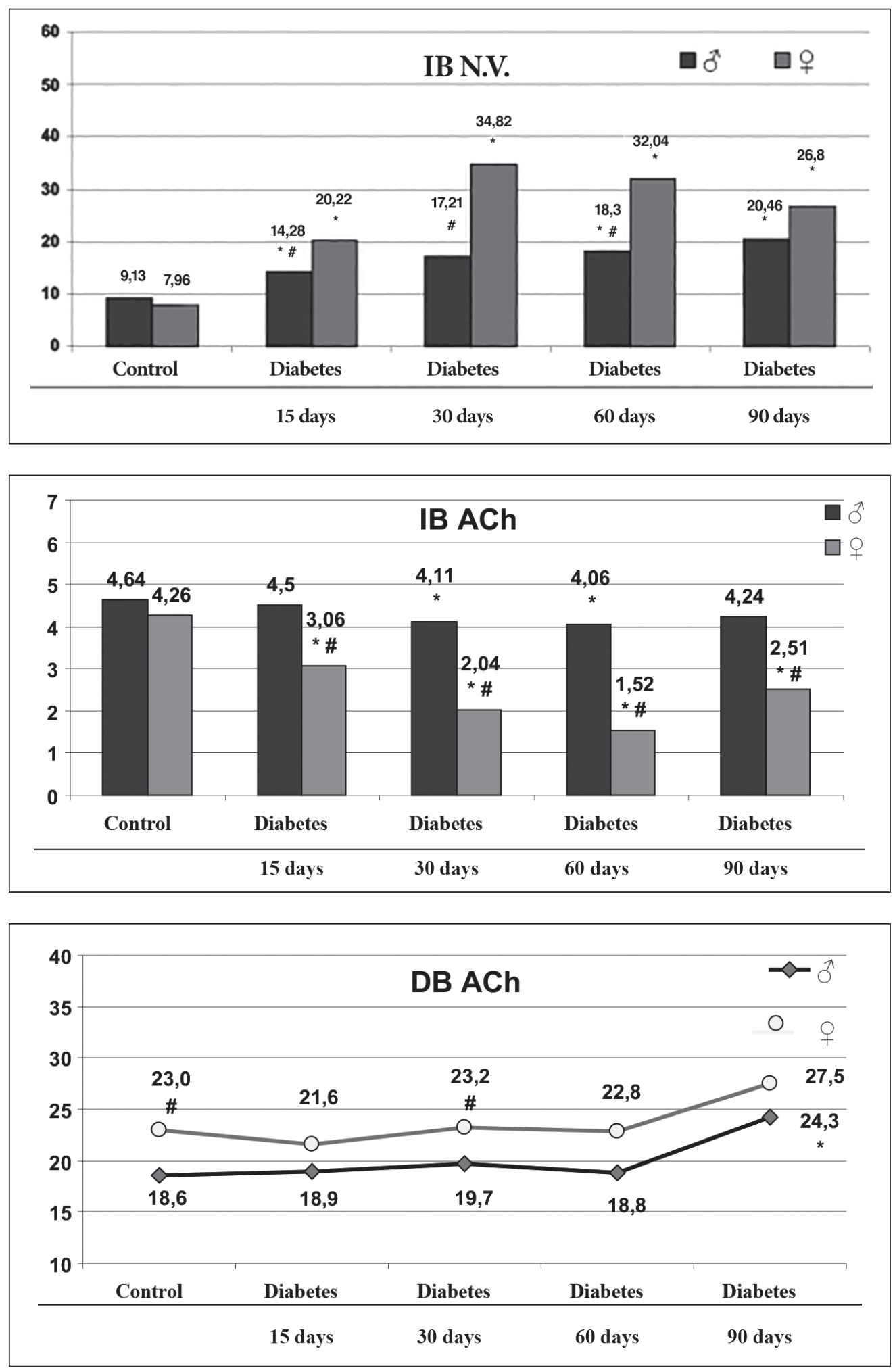

Fig. 1. Dynamics of bradycardia intensity that appeared with the electrical stimulation of the vagus nerve (IB N.V., abs. number, $\mathrm{n}=6$ ) in males and females in the development of streptozotocininduced diabetes.

Fig. 2. Dynamics of intensity of bradycardia after intravenous injection of acetylcholine (IB ACh, abs. number, $n=6$ ) in males and females in the development of streptozotocin-induced Diabetes.

Fig. 3. The dynamics of the value of the duration of bradycardia that began after intravenous injection of acetylcholine (DB ACh, sec., $\mathrm{n}=6$ ) in males and females in the development of streptozotocininduced Diabetes. and structural disorders in the myocardium with diabetes, depending on gender[12].

\section{THE AIM}

Find out the nature of the changes sensitivity of the heart to the cholinergic influences the development of diabetes depending on gender.

\section{MATERIALS AND METHODS}

Research of the heart reactions on electrical stimulation of the right vagus nerve allows to evaluate the quanta reserve of acetylcholine in the presynaptic section of vagus nerves and their ability to disentangle during receiving efferent impulse. These effects evaluated in terms of intensity vagus bradycardia (IB N.V.). The potency of heart answer on such impact largely depends on a sensitivity of postsynaptic 
cholinoreceptors. The experiment of the process was done by intravenous injection of acetylcholine and also estimated the largest intensity of bradycardia (IB ACh), which had the effects on heart. Also determined another indicator - the duration of that bradycardia effect (DB ACh). This figure depends on several components: first - the activity of enzymatic hydrolysis parasympathetic neurotransmitter into the synaptic cleft, and the second - the ability of cholinergic receptors for functional desensitization to protect sinus pacemakers from prolonged hyperpolarization.

\section{RESULTS AND DISCUSSION}

Taking into account that the autonomic nerve system is response for the development of diabetic cardiomiopatia and implemented at the level of the heart through the receptor system. For understanding the mechanism of that changes, their feasibility and effectiveness, the next stage of the research was to establish the character of the sensitivity of the cholinergic structures of the heart on stimulating effects.

The results are presented in figure 1 , show that electrical stimulation of the vagus nerve intensity of vagus bradycardia in males was $(9,13 \pm 0,12)$. After 15 days from the start of streptozotocin model of diabetes this figure increased to $(14,28 \pm 0,45)$. This difference was 1.6 times $(p<0.001)$. The established tendency showed a gradual increase in this indicator and was preserved in the future. After 30 days of the experiment, the figure that was $(17,21 \pm 0,78)$,and was higher then control number in 1.9 times $(\mathrm{p}<0.001)$.

After 60 days of experiment, vagal bradycardia intensity is equal $(18,30 \pm 0,56)$ and was higher than in control males for 2 times ( $\mathrm{p}<0.001)$. At the last stage of experiment, 90 days after the value of intensity vagus bradycardia in males was $(20,46 \pm 1,83)$ and was higher in comparing with the first one in 2.2 times $(\mathrm{p}<0.001)$.

In females the dynamic intensity of vagus bradycardia was brighter and had wavy character (fig. 1). So, the index in control animals was $(7,97 \pm 0,09)$, After 15 days from the first injection of streptozotocin, the number increased to $(20,22 \pm 0,83)$. This increment was 2.5 times $(p<0.001)$. After 30 days of experiment we could see the tendency how this index increased and and continued to be constant. The value of the intensity of vagus bradycardia increased to $(34,82 \pm 1,18)$ and dominated the control numbers in 4.4 times $(\mathrm{p}<0.001)$. In the future, the dynamics of this index showed a different character. There was a tendency to reduce it. As a result, the difference from the original index became smaller. So, after 60 days from the beginning of streptozotocin induced Diabetes, the value intensity of vagus bradycardia was $(32,04 \pm 0,96)$. The advantage above the controlled females during this period of experiment was 4 times $(\mathrm{p}<0.001)$.

After 90 days of experiment the value of that number reached $(26,80 \pm 2,74)$. It was higher just in 3.4 times ( $p$ $<0.001)$. This was the result of its reduction in comparison with the 30-th day of observation for $23 \%$ ( $\mathrm{p}<0.05$ ).

In comparison with the intensity of vagus bradycardia male and female, it showed not only a different character of dynamics. In males, the figure gradually declined in the development of diabetes and the intensity of dynamics was mild. Instead of females in the early stages of the experiment (15 and 30 days from the beginning of diabetes) significantly increased with the intensity of vagus bradycardia and had a tendency to be decreased .

The magnitude of this indicator of animals had not always changed. In particular, the control number was not significant different between males and females . After 15 days of diabetes intensity value of vagus bradycardia was higher in females than in males in 1.4 times $(\mathrm{p}<0.001)$. After 30 days this difference increased and amounted to 2 times ( $\mathrm{p}<0.001)$. After 60 days of diabetes it was 1.8 times $(\mathrm{p}<0.001)$ and after 90 days there were no difference between control numbers.

Analysis of the reaction of the heart to intravenous acetylcholine, showed one direction dynamics that revealed a decrease in this indicator (fig. 2). In the control group of males it was equal to $(4,64 \pm 0,17)$. After 15 days it was $(4,50 \pm 0,22)$. Significant differences with the control was not established $(\mathrm{p}<0.05)$.

After 30 days of the experiment, the intensity value of bradycardia, which happened because of intravenous acetylcholine injection, decreased to $(4,11 \pm 0,05)$. At this stage the difference was authentic, although reduction was only for $11 \%(\mathrm{p}<0.02)$. After 60 days it was $(4,06 \pm 0,14)$, lower than the original for $13 \%(\mathrm{p}<0.02)$, and 90 days, the value index $(4,24 \pm 0,47)$ significant differences of the control was not installed.

In females, these changes had similar direction, but substantial. In control animals the intensity value of bradycardia after intravenous injection of acetylcholine was $(4,26 \pm 0,14)$. After 15 days of streptozotocin injection,the value of the index has decreased to $(3,06 \pm 0,05)$, which was significant and was 1.5 times $(\mathrm{p}<0.001)$. After 30 days of experiment, this tendency continued. Reducing the rate to $(2,04 \pm 0,01)$ it was more significant and amounted to 2.3 times $(p<0.001)$. After 60 days from the beginning of diabetes, this figure was $(1,52 \pm 0,12)$, which was 3 times $(p<0.001)$ lower than the original figure. After 90 days, the value of intensity bradycardia occurred after intravenous injection of acetylcholine, which was $(2,51 \pm 0,12)$, was in 1.7 times $(\mathrm{p}<0.001)$ higher than in the previous stage of experiment, while remaining lower than in controls in 1.7 times $(\mathrm{p}<0.001)$.

Despite a similar dynamic test rate in males and females, due to its greater intensity in the last absolute values,differed at various stages of development of diabetes. The main feature of this difference was that in female intensity value of bradycardia, which corresponded heart to intravenous acetylcholine was significantly smaller. In the absence of such a difference in the control ( $p>0.05)$ After 15 days benefit rate of males was in 1.5 times $(\mathrm{p}<0.001)$ After 30 days -in 2 times $(\mathrm{p}<0.001)$ after 60 days -2.7 times $(\mathrm{p}<0.001)$ and 90 days -in 1.7 times. Reducing differences in the last stage of observation was the result statistically significant increase in this indicator compared to the previous phase observations in 1.7 times $(\mathrm{p}<0.001)$. 
Analysis of figures of bradycardia started with intravenous injection of acetylcholine, showed not so bright results as the dynamics of the two options described above (fig. 3). In males this value in control was $(18,6 \pm 0,3)$. After 15 days of the experiment, it was $(18,9 \pm 1,2)$, after 30 days of observation - $(19,7 \pm 1,0)$, after 60 days - $(18,8 \pm 1,7)$, and after 90 days - $(24,3 \pm 2,3)$. Despite different absolute values of its fluctuations throughout most of the observation period of the development of diabetes were within the statistical mistakes ( $p>0.05$ ).Especially on 15th day, 30th day and $60^{\text {th }}$ day of experiment. However, 90 days after the diabetes, the figure was significantly greater than the value of the control group for $30 \%(\mathrm{p}<0.05)$.

In females the value of duration of bradycardia, which began after intravenous injection of acetylcholine, also within the statistical error. In the control group this index was $(23,0 \pm 1,1)$.

After 15 days of streptozotocin model of diabetes, the index was $(21,6 \pm 1,3)$, after 30 days - $(23,2 \pm 0,6)$, after 60 days - $(22,8 \pm 1,9)$, and 90 days after the experiment - $(27,5$ $\pm 3,0)$. Significant differences relative measure of control animals that was not on any of the stages of development of diabetes $(\mathrm{p}>0.05)$.

The comparison of animals with different sexes showed that in the control group animals duration of bradycardia, which corresponded to the female heart to intravenous acetylcholine was significantly higher than in males. The difference was $24 \%$ ( $p<0.01)$. After 15 days from the beginning of diabetes, detected difference of absolute values was within the statistical error ( $p>0.05)$. After 30 days of experiment,the dynamics of the duration of bradycardia showed that the heart of animals with intravenous acetylcholine in female figure was bigger than the males. The advantage was $18 \%$ and was authentic $(\mathrm{p}<0.02)$. After 60 and 90 days of experiment,there was not a significant differences for this indicator. between males and females. The nature of the dynamics of this index was similar in animals of both sexes.

In general, described changes in heart rate and variation cardiointervalmetria indicators showed that the development of streptozotocin-induced diabetes in males and females characterized by different heart reactions and autonomic nerve system.

Males in the early stages of experiment had a bradycardia. These changes were recorded after 15 and 30 days after streptozotocin injection. The reaction of the autonomic nerve system showed a decrease adrenergic control of the heart thtough the humoral canal, confirmed an increase in module. The absence of amplitude dynamics of module testified maintain control heart rhythm this link autonomic regulation, which implements the functional effects through the nervous canal. The gradual increase of variation magnitude indicative of moderate cardio cholinergic activation level of autonomic regulation that contributed to its general dominance in the formation of heart rate and a decrease in confirmed integral indicators - index of stress regulatory mechanisms, autonomic balance index, vegetation index rhythm and rate adequacy of regulatory processes.
Despite the fact that the progression of diabetes in males led to the development of tachycardia, heart rate regulation had signs of further growth and a significant level of dominance cholinergic autonomic regulation, which showed a decrease in the above integrated indicators. These changes were the result of the significant increase in the participation of the vagus nerve in the formation of heart rate, as evidenced by a progressive increase cardio variations scope and limitations adrenergic implemented through the nerve canal (confirmed decrease in the amplitude of module).

State of cholinergic receptors also experienced change of heart, the essence of which was strengthening the heart response to electrical stimulation of the vagus nerve and slight intensity reduction reaction of the heart to intravenous acetylcholine. This indicated facilitated the release the quanta of acetylcholine from the presynaptic process against a slight decrease in the reactivity of postsynaptic cholinergic receptors. Synergetic growth of cardio intervals, despite an increase in heart rate, demonstrated the dominant role of cholinergic mechanisms in the heart.

In female rats, the development of diabetes in early stages called tachycardia. Regulation of heart rate with the variation according cardiointervalmetria is characterized by a decrease in amplitude and increase and decrease of module, that cause a significant increase in adrenergic part autonomic nerve system on the formation of the heart rhythm. The increase of the index of tension and the adequancy of regulation processes reflects the dominance of the early stage of diabetes adrenergic mechanisms of the heart.

The reduction of heart rate began with a growth of module and reducing the amplitude of module that demonstrated significant limitation of adrenergic activity level in autonomic regulation of the heart. Cholinergic activity level under these conditions was growing moderately, as evidenced not only a slow increase in the cardio intervals, but smaller than in males. The gradual reduction of integrated indicators - index of stress regulatory mechanisms, autonomic balance index, vegetation index rhythm and rate adequacy of regulatory processes collectively reflect the dominant role of cholinergic autonomic nerve system in the formation of heart rhythm. Despite of bradycardia, its contribution to the formation of heart rate was similar to that in males.

\section{CONCLUSIONS}

The changes that was described above in females with diabetes occurred in the more substantial than in males, heart response to electrical stimulation of the vagus nerve. This response of the heart to intravenous acetylcholine, significantly decreased. Totally, it demonstrated the accumulation and slight excretion of acetylcholine in the presynaptic membrane.

Therefore, we can make the following intermediate conclusion:

1. The development of streptozotocin induced diabetes causes a cardio rhythm disordes and problems with vegetative regulation of rats' heart, which character 
depends mainly on gender. In males, there is a moderate level reducing of adrenergic autonomic nervous system in regulating the heart and significant increase of cholinergic. In females, the development of diabetes is less intensive, than in males, and decreasing of activity in adrenergic and increasing of cholinergic links in the formation of heart rhythm is different too.

2. The progress of diabetic cardiomyopathy is more significant in female rats compared with males, strengthening the response of heart to stimulate the vagus nerve and its reduction by exogenous acetylcholine, that shows the increase of reserve in presynaptic neurotransmitter parasympathetic mediator of the vagus nerve and basically causes a bradycardia, due to deeper than in males reducing of the sensitivity of postsynaptic cholinergic receptors.

\section{REFERENCES}

1. Acbar S. Bellary S., Griffiths H.R., Dietary antioxidant interventions in type 2 diabetes patients: a meta-analysis Brit. J. Diabetol. Vasc. Dis. 2011;11(2):62-68.

2. Becher P.M., Lindner D., Frohlich M., et al. Assessment of cardiac inflammation and remodeling during the development of streptozotocininduced diabetic cardiomyopathy in vivo: A time course analysis, Int. J. Mol. Med. 32(1)158-164.

3. Billimoria F.R., Katyare S.S., Patel S.P., Insulin status differentially affects energy transduction in cardiac mitochondria from male and female rats, Diabetes Obes. Metab. 2006;8(1):67-74.

4. Goyal B.R., Mehta A.A., Diabetic cardiomyopathy: pathophysiological mechanisms and cardiac dysfuntion, Hum. Exp. Toxicol. 2013;32(6):571-590.

5. Lindmark S., Lonn L., Wiklund U., [et al.] Dysregulation of the autonomic nervous system an be a link between visceral adiposity and insulin resistance, Obes. Res. 2005;13(4):717-728.

6. Mabe A.M., Hoover D.B., Remodeling of cardiac cholinergic innervation and control of heart rate in mice with streptozotocin-induced diabetes, Auton. Neurosci. 2011;162(1-2):24-31.

7. Maser R.E., Mitchell B.D., Vinik A.I., [et al.], The association between cardiovascular autonomic neuropathy and mortality in individuals with diabetes, A meta-analysis, Diabetes Care. 2003;26:1895-901.

8. Pop-Busui R., Cardiac Autonomic Neuropathy in Diabetes. A clinical perspective Diabetes care. 2010;33(2):434-441.

9. Uchiyama Y., Nakao S., Asai T., Shingu K., A case of atropine-resistant bradycardia in atient on long-term lithium medication Masui. 2001:50(11):1229-1231.
10. Wang C., Li J., Lv X.et al. Ameliorative effect of berberine on endothelial dysfunction in diabetic rats induced by high-fat diet and streptozotocin, Eur J Pharmacol. 2009;620(1-3):131-137.

11. Witte D.R., Tesfaye S, Chaturvedi N. et al. Risk factors for cardiac autonomic neuropathy in type 1 diabetes mellitus, Diabetologia. 2005: 48(1):164-171.

12. Yang H., Wright J.R., Human beta cells are exceedingly resistant to streptozotocin in vivo, Endocrinology. 2002:143(7)2491-2495.

This article is a fragment of the complex scientific research work of the educational-scientific institute of modeling and analysis of pathological processes of the State Higher Educational Institution «Ternopil State Medical University named after I.Ya. Gorbachevsky Ministry of Health of Ukraine "» Medical regularities and information models of the course of pathological processes under various functional conditions and their correction «(state registration number 0110U001937).

\section{ORCID and contributionship:}

Orysia O. Bandrivska - 0000-0002-3274-1781 B,C,D

Vitalij M. Mykhailiuk - 0000-0002-9877-0112 ${ }^{F}$

Iryna A. Mykhailiuk - 0000-0002-2088-5075 ${ }^{\mathrm{E}}$

Natalia V. Malko - 0000-0002-3272-1836 ${ }^{A}$

\section{Conflicts of interest:}

Authors declare no conflict of interest.

\section{CORRESPONDING AUTHOR Orysia 0. Bandrivska}

I. Horbachevsky Ternopil State Medical University

Brygadna, 14a. apt 86, 46012, Ternopil, Ukraine

tel: +380973047399

e-mail: bandrivska.83@gmail.com

Received: 21.05 .2019

Accepted: 30.12 .2019

A - Work concept and design, B - Data collection and analysis, C - Responsibility for statistical analysis, D-Writing the article, $\mathbf{E}-$ Critical review, $\mathbf{F}$ - Final approval of the article 